\title{
Investigating Behavioral Differences between loT Malware via Function Call Sequence Graphs
}

\author{
Reo Kawasoe \\ Kyushu University \\ kawasoe@me.inf.kyushu-u.ac.jp
}

\author{
Chansu Han \\ National Institute of Information and \\ Communications Technology, \\ Kyushu University
}

\author{
Ryoichi Isawa \\ National Institute of Information and \\ Communications Technology
}

\author{
Takeshi Takahashi \\ National Institute of Information and \\ Communications Technology
}

\author{
Jun'ichi Takeuchi \\ Kyushu University
}

\begin{abstract}
IoT malware that infects IoT devices is rampant. Most IoT malware variants are generated by changing various behaviors such as an attack method based on existing malware families. Nearly all antivirus software only identifies the malware family's name; thus, we cannot acquire further details about differences between malware behaviors. In this paper, we propose a graph-based method for confirming differences in malware behaviors and investigating the actual conditions of malware variants. The proposed method first extracts a sequence of function calls from a binary file of malware and represents the sequence to a directed graph, which we refer to as a function call sequence graph (FCSG). Next, the method automatically checks if the FCSG matches signature-FCSGs, which are manually generated as small-scale FCSGs representing malicious behaviors of known malware such as a function of attacks and network scans. To demonstrate the usability of our proposed method, we applied the proposed method to 24,126 in-the-wild IoT malware specimens and investigated the existence of specimens with mixed behaviors from multiple malware families or were specialized for some attacking behaviors.
\end{abstract}

\section{CCS CONCEPTS}

- Security and privacy $\rightarrow$ Malware and its mitigation;

\section{KEYWORDS}

IoT malware, static analysis, function call sequence graph, signature match, behavioral difference

\section{ACM Reference Format:}

Reo Kawasoe, Chansu Han, Ryoichi Isawa, Takeshi Takahashi, and Jun'ichi Takeuchi. 2021. Investigating Behavioral Differences between IoT Malware, via Function Call Sequence Graphs. In The 36th ACM/SIGAPP Symposium on Applied Computing (SAC '21), March 22-26, 2021, Virtual Event, Republic of Korea. ACM, New York, NY, USA, Article 4, 9 pages. https://doi.org/10. $1145 / 3412841.3442041$

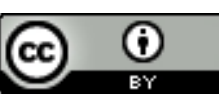

This work is licensed under a Creative Commons Attribution International 4.0 License. SAC'21, March 22-March 26, 2021, Gwangju, South Korea

(C) 2021 Copyright held by the owner/author(s).

ACM ISBN 978-1-4503-8104-8/21/03.

https://doi.org/10.1145/3412841.3442041

\section{INTRODUCTION}

With the widespread use of IoT devices, IoT malware that infects vulnerable IoT devices is spreading. Mirai, which is a family of IoT malware, has formed a large-scale botnet and caused considerable damage, including the world's largest 623 Gbps DDoS (Distributed Denial-of-Service) attack [2]. Malware whose source code has been released or leaked, e.g., Mirai and Bashlite, has become more easily exploited and causes different variants to proliferate. More than 70,000 new IoT malware were identified in the first quarter of 2020 , and this number has been increasing year over year [14]. Therefore, accurate analysis of malware functionality and quick responses are required to prevent damage.

Reusing a code base or sharing code logic is common in malware [19]. In addition, malware variants evolve as attackers gradually change their behaviors. Thus, it is important for malware variants to see what behaviors have been added or removed. Antivirus software analyzes malware specimens to determine which malware family a specimen belongs to. However, it is very difficult to identify what behaviors each malware specimen can invoke and what behavioral differences exist between malware specimens even in an identical malware family. For example, a specimen identified as Mirai could send UDP packets for DDoS attacks, whereas another specimen of Mirai would not possess that functionality.

In a previous study, Iwamoto et al. [9] analyzed Windows malware statically, generated directed graphs by extracting application programming interface (API) calls, and then clustered malware by calculating the graph similarity between malware specimens. Their method draws an API call graph from static analysis and visualizes API call relationships; however, they only focused on clustering. That is, their method is insufficient to know specific behaviors of each malware in a cluster. In addition, they did not consider userdefined functions and the effects of other environmental factors, e.g., instruction set architecture (ISA) and compilers. We have set out to expand the visualization of malware specimens' behavior in API call graphs and consider a method to identify behavioral differences using signatures rather than a malware clustering technique.

To investigate the actual conditions of malware, especially IoT malware, we attempt to automatically estimate behavioral differences between large-scale malware specimens by focusing on which functions (e.g., open, read, printf, and close in C programming) are called and their sequence. Some of those function calls are used to implement specific behaviors of malware. Here, a behavioral difference refers to changes in attack types and malware behaviors. 
In this paper, we propose a method to generate a function-callsequence graph (FCSG) that considers both API names and names of called user-defined functions from the disassembled code of malware specimens. In addition, we implement a method to avoid environmental problems in IoT malware, e.g., ISA and compilers, by applying normalization on function names or removing nodes in FCSGs. Next, we generate the FCSGs from the disassembly result or the released source code of a specific IoT malware family, and the characteristic behaviors of the malware are created manually as signature-FCSGs. When a signature-FCSG is matched to the FCSGs of a malware specimen, the malware specimen can be considered to have a behavior that corresponds to the signature-FCSG.

The proposed method is used to specify malicious behaviors of malware binaries captured by honeypot systems for investigating functional behaviors between malware, assuming that the honeypot systems do not capture any benign software (i.e., the proposed method does not target any benign software in this paper). Also, an FCSG and a signature-FCSG can handle malware on different ISAs because it focuses only on function calls. Moreover, an analyst can confirm function calls briefly, and signatures can be created easily from the source code or disassembled code.

Contributions: To the best of our knowledge, this is the first work to extract behaviors contained in IoT malware specimens in a graph, confirm the behavioral differences between specimens using graph-based signatures, and discover specimens containing activities from multiple malware families. In this study, we used FCSGs to investigate behaviors of 24,126 in-the-wild IoT malware specimens running on Linux of IoT devices and confirmed that ISA-independent signature matching is possible. Our primary contributions are summarized as follows.

- We propose generating a FCSG from the disassembled code of a malware specimen and estimating the behavioral differences among large-scale malware specimens regardless of ISAs or malware families (Sections 3 and 4).

- We identify mixed variants with multiple malware family features that match some signature-FCSGs generated from the source code of different malware families (Section 5.2.1).

- We identify malware variants that may have been removed from some behaviors and specialized in some attacking behaviors (Section 5.2.2).

\section{PRELIMINARIES}

Here, we explain some important elements; instruction set architecture and disassemble by IDA Pro.

\subsection{Instruction Set Architecture}

There are multiple CPU types (e.g., ARM, MIPS, and Intel x86) used in IoT devices, and each CPU has its own unique instruction set architecture (ISA). This fact makes us difficult to identify the behavior of IoT malware as follows.

Binary files with different ISAs cannot be executed on the same Linux OS (e.g., a specimen of ARM does not run on Linux OS built for MIPS); therefore, many IoT malware prepares binary files compiled for multiple ISAs in advance and executes the binary that is suitable for a target IoT device to be infected. This results in that different binaries can be generated by compilers even from the same source code if the target ISA is distinct.

Such malware binaries pose this challenge: how well can we identify a specific behavior (e.g., sending UDP packets for DDoS attacks) shared between given binaries with different ISAs? If we simply compare those binaries byte by byte, this result is to show that the malware binaries greatly differ from each other due to the different ISAs, whether or not they are generated by reusing any part of the same source code. Identifying behaviors of IoT malware directly leads to capturing behavioral differences between IoT malware, and so we tackle this problem in this paper with a graph-based method focusing on function calls, which is proposed in Section 3.

Note that we use a reverse engineering tool to extract function calls without executing malware specimens (i.e., in a static analysis manner) for our method. We can also choose dynamic analysis; however, there are some major drawbacks of dynamic analysis. One is that executing each specimen is time consuming, and another drawback is that IoT malware would not reveal malicious behaviors unless they receive commands through their C2 (Command and Control) servers.

\subsection{Disassemble by IDA Pro}

IDA Pro [8] is a reverse-engineering software tool working for multiple ISAs (e.g., ARM and MIPS). This tool can disassemble a binary file to provide its basic blocks, where a basic block of a binary file means a set of multiple machine codes that are divided by branch and return instructions. The tool also provides a flow chart (i.e., the control flow) of the processing order of the basic blocks.

With IDA Pro, we can obtain function calls in the processing order from that control flow because basic blocks include function calls embedded in a binary file and they are sorted in the processing order. Those function calls are used for generating the FCSGs introduced in Section 3.2. In addition, IDA Pro shows good performance against IoT malware [5], which also supports us to choose IDA Pro as the reverse-engineering tool we use in this study.

\section{PROPOSED METHOD}

In this section, we propose a method for identifying behaviors of IoT malware. This leads to investigating behavioral differences between IoT malware as is demonstrated in Section 5.

\subsection{Overview}

We assume that a specific behavior of IoT malware is achieved by a set of functions including system calls (e.g., socket, bind, listen, and exec). Examples of such behaviors include accessing to a C2 (Command and Control) server and executing a Linux command.

Figure 1 shows an overview of the proposed method for identifying behaviors of a given specimen. Our method focuses on the FCSG (Function Call Sequence Graph) explained in Section 3.2 in detail. Intuitively, the FCSG is a directed graph of functions with the processing order. Given a specimen, the whole binary file can be represented as an FCSG, and a specific behavior is encapsulated by a sub-graph in the FCSG. The key idea behind our method is to previously extract sub-graphs corresponding to behaviors as signatures. 


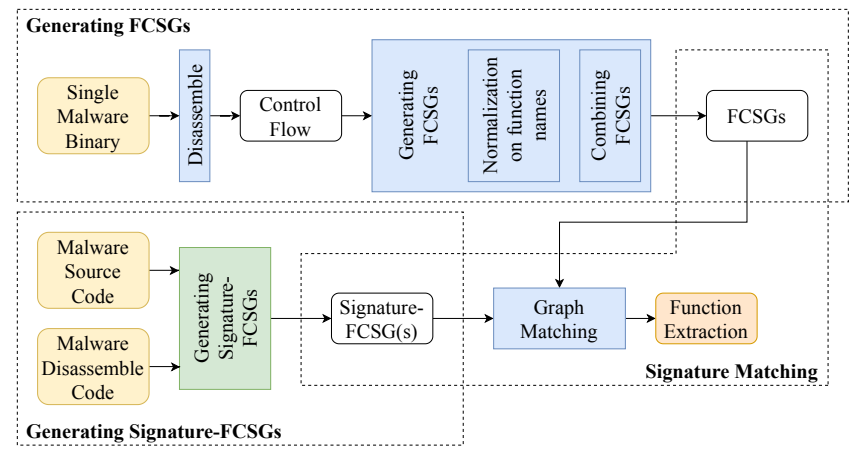

Figure 1: Overview of proposed approach (yellow: inputs; orange: outputs; blue: automatic process; green: manual process)

For identification of behaviors, we manually create signatures (as Generating Signature-FCSGs in Fig. 1). We then automatically generate FCSGs of target malware specimens with tools (as Generating $F C S G s$ ). Finally, we also automatically conduct a signature matching against those specimens to be investigated (as Signature Matching). If a signature-FCSG is matched to an FCSG of a specimen as a sub-graph, this specimen is considered to perform the behavior corresponding to the signature.

There is a problem of the ISA differences that we aim to solve, as is explained in Section 2.1. We have to match a signature with IoT malware specimens even if they are binaries with different ISAs. The signature of FCSG can absorb the ISA differences because it only focuses on functions as nodes of FCSG. This is based on the fact that those functions will be called in the processing order predefined in source code without depending on ISAs, and so the FCSG and the signature are robust against ISA differences and will work well.

For details, Generating FCSGs is explained in Section 3.2, and Generating Signature-FCSGs and Graph Match are explained in Section 3.3.

\subsection{Function Call Sequence Graph}

The FCSG, a directed graph representing the sequence of function calls, is extracted from a binary file as follows. A binary file is disassembled to obtain its disassembly code, and all function calls invoked by the binary are recognized. For each function (e.g., main and get_random), the control flow of the basic blocks is created. Given a control flow, the corresponding FCSG is then generated by extracting function calls from the control flow with preserving the processing order. In FCSGs, a node is a function call labeled with its function name, and a directed edge is linked from a function to next function to be called according to the control flow. The root node of an FCSG is the function name for which the corresponding control flow is created (e.g., main and get_random).

For better understanding FCSG, Fig. 2 shows the control flow of main function of Program 1 compiled by the GCC compiler for ARM (named arm-linux-gnueabi-gcc v9.3.0), and Fig. 3 shows that of get_random function of Program 1. The corresponding FCSGs to main and get_random are shown in Fig. 4. As get_random $\rightarrow$

\section{Program 1: Sample program}
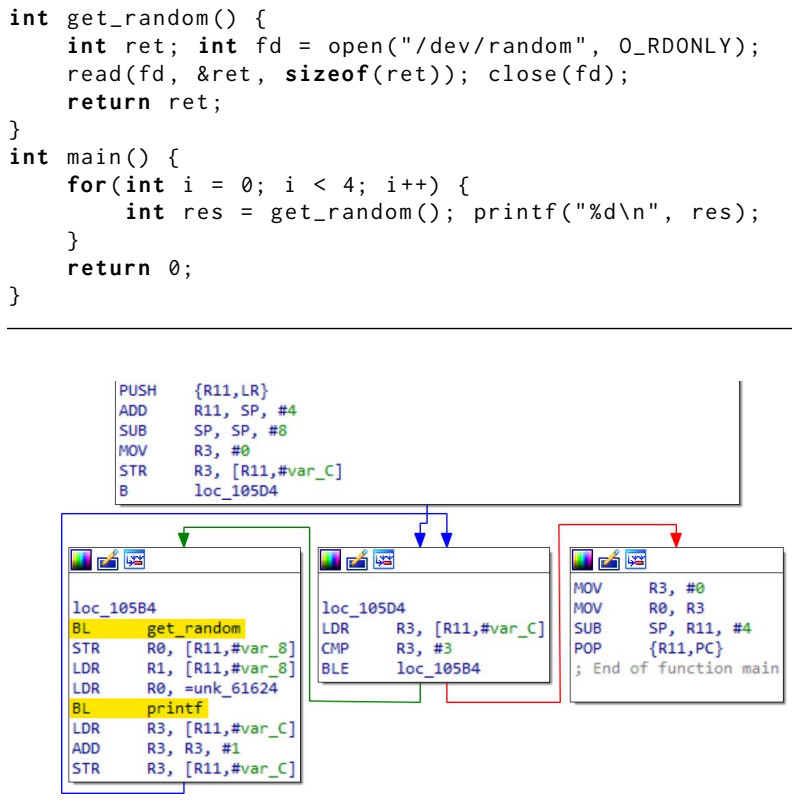

Figure 2: The control flow of main function from ARM binary (in Program 1)

printf, a directed edge is linked from a function to next function to be called. The root node main is also linked to get_random, which is called first in the control flow of main.

An FCSG is combined with another FCSG if any node in an FCSG is the root node in another FCSG. For example, in Fig. 4, get_random node in the left FCSG is the root node in the right FCSG, and then those FCSGs are combined to the united FCSG shown in Fig. 5. In this manner, the FCSGs for all functions are combined as a united FCSG of a specimen, except for standard functions (e.g., open and read in C) including Linux system calls. The FCSG for a standard function could be also generated from the functions that consist of that standard function; however, we do not generate any FCSGs of standard functions. This is because standard functions do not conduct malicious tasks by themselves. We only generate FCSGs of use-defined functions and combine them.

As a case study for showing robustness of FCSG against different ISAs, Figures 7 and 8 show the control flows obtained with IDA Pro after compiling Program 1 by the GCC compiler for MIPS (mipslinux-gnu-gcc v9.3.0). Comparing Fig. 7 (compiled for MIPS) with Fig. 2 (complied for ARM), it can be seen that the structures of basic blocks of main are quite different. On the contrary, there is no difference in the sequence of function calls. This can be also seen comparing Fig. 3 with Fig. 8. By using FCSGs, it is possible to extract the behavior even in different ISAs.

\subsection{Signature Matching}

Signatures of FCSG are manually generated as follows. We obtain the source code of an IoT malware specimen or the disassembly code. We then analyze the codes to learn specific behaviors of the 


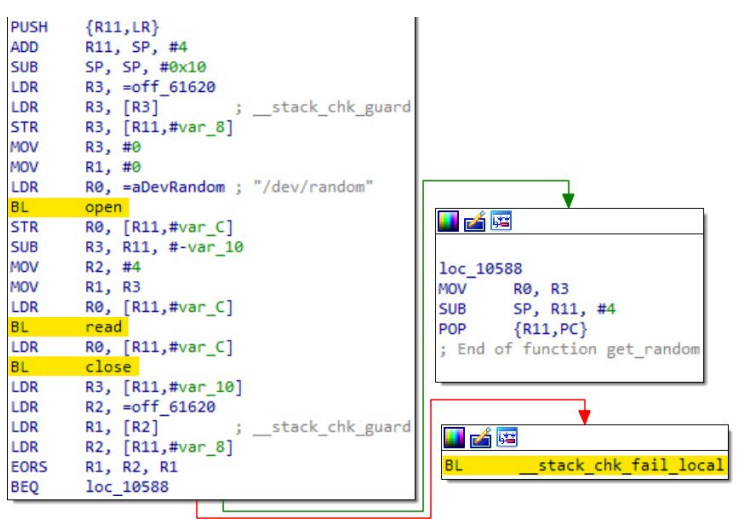

Figure 3: The control flow of get_random function from ARM binary (in Program 1)

specimen and the part of the code corresponding to each behavior. After that we generate a FCSG from the corresponding part of the code for a behavior. We call this FCSG a signature-FCSG for that behavior. For identifying behaviors of a given specimen, we check if a signature-FCSG is matched to the united FCSG of the specimen as a sub-graph. If they match, we determine that the specimen has the behavior corresponding to the matched signature. For example, when the signature-FCSG (open $\rightarrow$ read $\rightarrow$ close) in Fig. 6 is applied the combined FCSG in Fig. 5, the combined FCSG's dashed nodes are matched. This signature represents the reading of a file.

IDA Pro cannot obtain function names from specimens in the following case: Mirai specimens may call user-defined functions through a function-descriptor table previously embedded by malware authors in the specimens. For this, the specimen first obtains the address of a function from the descriptor table. The obtained address is then stored in a CPU register (e.g., r8 in Intel x86_64), and via this register the function is called (e.g., call $r 8$ ). In this case, IDA Pro just identifies the register name used for use-defined function calls. To treat with this problem, we take the register name (e.g., r8) as the name of a node in FCSGs to generate FCSGs of the specimen. Because IDA Pro does not identify which function is called via the CPU register, we do not combine FCSGs based on any register names.

\section{IMPLEMENTATION}

This section introduces an implementation of the proposed method.

\subsection{Outline of Proposed Method}

Algorithm 1 describes instructions in pseudo code that are followed after manually generating signature-FCSGs. In the algorithm, function GenerateFCSGs takes as input a malware binary $M$ to output FCSGs of $M$, and function SignatureMatching conducts signature matching against $M$ with each of the input signature-FCSGs $\left[S_{1}, S_{2}, \ldots,\right]$. The output of Algorithm 1 is a signature-match vector $V$. This vector represents which signatures are matched to $M$, and the malware is then considered to have the specific behaviors corresponding to the matched signatures.

In the rest of this section, Section 4.2 explains how we manually generated signature-FCSGs. Sections 4.3 and 4.4 explain functions

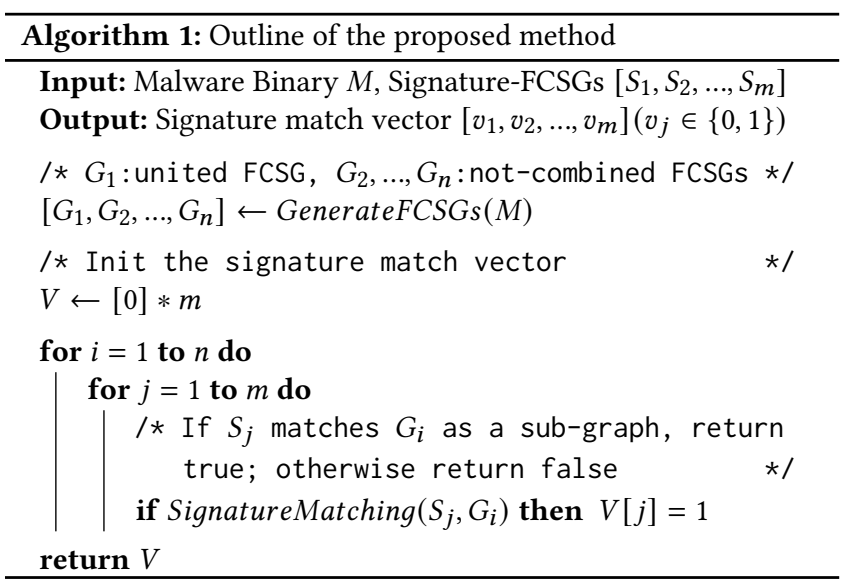

Table 1: List of created Bashlite signatures

\begin{tabular}{ll}
\hline Signature Name & Behavior \\
\hline \hline access_to_c2 & Check if the C2 server is accessible \\
\hline echo_build_info & $\begin{array}{l}\text { Send its own binary information } \\
\text { to the C2 server }\end{array}$ \\
\hline copy_process & Process replication \\
\hline ping_pong & $\begin{array}{l}\text { Reply 'PONG' when 'PING' is sent } \\
\text { from the C2 server }\end{array}$ \\
\hline exec_command & $\begin{array}{l}\text { Execute the command sent } \\
\text { from the C2 server }\end{array}$ \\
\hline get_parameter & Get the parameters sent from the C2 server \\
\hline get_ip & Get the IP address of the current device \\
\hline get_mac & Get the MAC address of the current device \\
\hline get_random_str & Generate a random string \\
\hline sendHOLD & Perform a HOLD attack \\
\hline sendJUNK & Perform a JUNK attack \\
\hline sendTCP & Perform a TCP attack \\
\hline sendUDP & Perform a UDP attack \\
\hline
\end{tabular}

GenerateFCSGs and SignatureMatching in detail, respectively. Those functions are automated with software tools and scripts.

\subsection{Generating Signature-FCSGs}

In this study, we select Mirai and Bashlite because they are very popular IoT malware samples and the source code of theirs is often reused. Their released source codes can be download from GitHub $[1,10]$. After obtaining the source code, we selected some basic behavior and behavior for attacking. We then generated a signature based on the file name, program structure, function name, and comments. Consequently, we generated 13 signatures for Bashlite and 15 signatures for Mirai, and these signatures are listed in Tables 1 and 2, respectively. The Bashlite source code comprises a single file, and the Mirai source code is divided into several files; thus, regarding Mirai's signature in Table 2, the file name is listed prior to the signature name. 

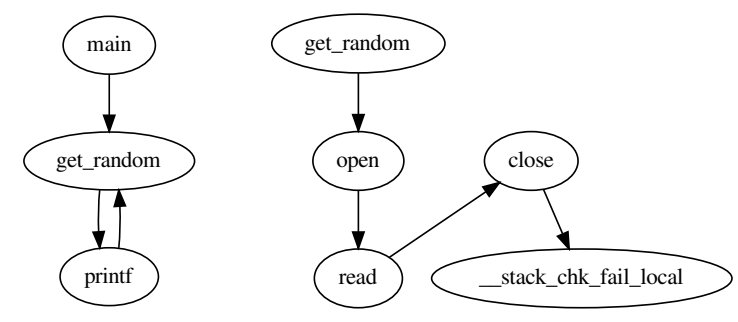

Figure 4: FCSGs for each control flow (in Fig. 2 and Fig. 3)

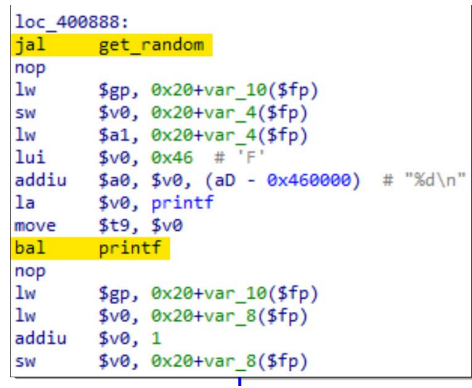

Figure 7: The partial control flow of main function from MIPS binary (in Program 1)

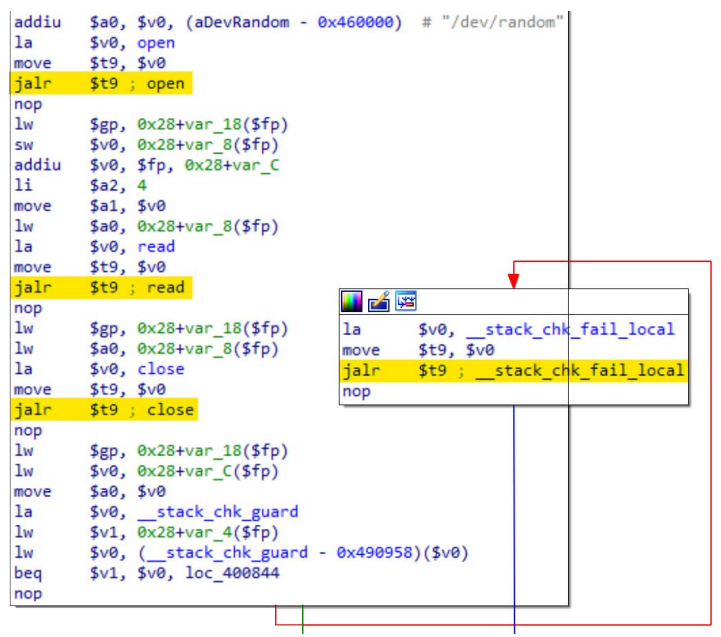

Figure 8: The partial control flow of get_random function from MIPS binary (in Program 1)

\subsection{GenerateFCSGs}

4.3.1 Generating FCSGs. As a plug-in of IDA Pro 7.4, we create a Python script to retrieve the control flow for each function and extract function call sequences from the control flow. This script searches the basic block in the control flow while considering branch instructions, and it finds call instructions. If the instruction calls a function name, the script records the called function
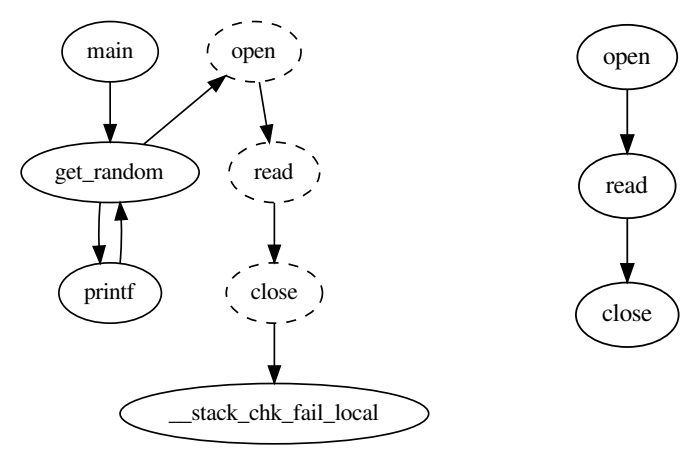

Figure 5: Combined FCSG from Fig. 4 Figure 6: Sample based on main function signature-FCSG

Table 2: List of created Mirai signatures

\begin{tabular}{|c|c|}
\hline (File name) Signature Name & Behavior \\
\hline (main) init_argcheck & $\begin{array}{l}\text { Check command line } \\
\text { arguments }\end{array}$ \\
\hline (main) init_hide_argv0 & $\begin{array}{l}\text { Hide command line } \\
\text { arguments }\end{array}$ \\
\hline (main) init_hide_pname & Hide process name \\
\hline (main) init_print_system_exec & $\begin{array}{l}\text { Get other running } \\
\text { processes }\end{array}$ \\
\hline (main) initializer & $\begin{array}{l}\text { Various initialization } \\
\text { processes }\end{array}$ \\
\hline (main) init_connect & $\begin{array}{l}\text { Establish a connection } \\
\text { to the } \mathrm{C} 2 \text { server }\end{array}$ \\
\hline (scanner) scanner_init_init & $\begin{array}{l}\text { Initialization of } \\
\text { other device scanners }\end{array}$ \\
\hline (scanner) scanner_init_main & $\begin{array}{l}\text { Execution of } \\
\text { other device scanners }\end{array}$ \\
\hline (attack) attack_parse & Get attack parameters \\
\hline (attack) attack_start & $\begin{array}{l}\text { Create a process } \\
\text { and start an attack }\end{array}$ \\
\hline (attack) add_attack & $\begin{array}{l}\text { Registration of } \\
\text { attack function }\end{array}$ \\
\hline (attack_app) attack_app_http & Attack on HTTP \\
\hline (attack_app) attack_app_cfnull & $\begin{array}{l}\text { Attack on HTTP } \\
\text { (large POST) }\end{array}$ \\
\hline (attack_gre) attack_gre_ip & Attack on GRE IP \\
\hline (attack_gre) attack_gre_eth & Attack on GRE Ethernet \\
\hline
\end{tabular}

name with the function name called immediately before in the same control flow. This script targets ISAs of Intel x86_64, x86, ARM MC 68000, MIPS, and Sparc, and it considers call, BL, bal, jal, jalr, and bsr. 1 as call instructions.

Next, we use Python 3.8 and NetworkX [6] 2.4 to generate a directed graph from function call sequences obtained by the script. Note that some function names are affected by fluctuations in notation and evident compiler influence; therefore the following processing is required.

4.3.2 Normalization on function names. When combining FCSGs, the label of nodes (i.e., function names) are used to match a node in a FCSG and the root node in another FCSG. Some compilers add 
Table 3: Breakdown of dataset by ISA and malware family

\begin{tabular}{c||cccccc||c}
\hline \multirow{2}{*}{$\begin{array}{c}\text { Malware } \\
\text { family }\end{array}$} & x64 & ARM & x86 & $\begin{array}{c}\text { MC } \\
68000\end{array}$ & MIPS & Sparc & Total \\
\hline \hline Bashlite & 2154 & 4871 & 4468 & 2069 & 4431 & 2033 & 20026 \\
\hline Mirai & 1 & 56 & & & 64 & 1 & 122 \\
\hline Tsunami & 49 & 110 & 94 & 44 & 118 & 38 & 453 \\
\hline Lightaidra & & 1 & & 3 & & & 4 \\
\hline SINGLETON & 1 & & & 1 & 3 & & 5 \\
\hline NaN & 372 & 909 & 729 & 365 & 795 & 346 & 3516 \\
\hline \hline Total & 2577 & 5947 & 5291 & 2482 & 5411 & 2418 & 24126 \\
\hline
\end{tabular}

specific prefixes to the names of standard library functions prior to generating binaries; therefore, to absorb the differences between compilers, we remove the beginning part of the function name. If the specific prefix containing underscore exists (such as _GI_, libc and_uClibc_), it is removed.

We then delete the following nodes (i.e., functions) from malware's FCSGs. ARM-based CPUs do not support the modulo operation at the machine language level. If a modulo operation exists in the source code, it is replaced with the modsi 3 function, which obtains similar operational results using a compiler for ARM. Second, when initializing an array, the memset function may or may not be used. Finally, the compiler adds the _-_errno_location function so the program can handle the error. Thus, all modsi3, memset, and _-_errno_location functions are deleted.

4.3.3 Combining FCSGs. We define user-defined functions as functions that are not listed in the man page provided by the JM Project [11]. However, in some Mirai malware, there are some functions that are called by the functions' address, not functions' name. Therefore, we combined FCSGs as much as possible; however, we also attempted signature matching for FCSGs that could not be combined.

\subsection{SignatureMatching}

We adopt the VF2 [3] graph matching algorithm, which is implemented on NetworkX. By default, the VF2 algorithm only checks a graph's shape; however, it is possible to check matching node names by defining a feasibility rule. Some compilers change the name of functions (e.g., strstr to strstr_r or _strstr). To treat such functions as the same function (e.g., strstr and _strstr are treated as the same function), we define the following feasibility rule: after unifying function names to the lowercase, nodes are matched if the function name on the signature side is partially included in the specimen's FCSGs.

\section{INVESTIGATION}

In this section, we show the results obtained by our investigation with the proposed method. It was conducted against the dataset containing in-the-wild IoT malware. This section begins with the outline of our dataset, followed by the discussion on the investigation results with some interesting findings.
Table 4: Order and size of Signature-FCSGs and FCSGs

\begin{tabular}{|c|c|c|c|c|c|c|}
\hline & & & Min. & Max. & Avg. & Std. \\
\hline \multirow{4}{*}{ 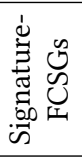 } & \multirow{2}{*}{ Bashlite } & order & 2 & 7 & 3.9 & 1.5 \\
\hline & & size & 1 & 7 & 3.5 & 1.6 \\
\hline & \multirow{2}{*}{ Mirai } & order & 2 & 14 & 6.0 & 4.0 \\
\hline & & size & 1 & 21 & 6.5 & 6.0 \\
\hline \multirow{6}{*}{ 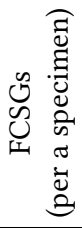 } & \multirow{2}{*}{ All } & order & 125 & 3534 & 538.2 & 189.1 \\
\hline & & size & 255 & 6723 & 949.6 & 243.6 \\
\hline & \multirow{2}{*}{ Bashlite } & order & 125 & 2599 & 530.6 & 178.4 \\
\hline & & size & 257 & 3753 & 944.1 & 230.3 \\
\hline & & order & 308 & 3534 & 556.7 & 302.3 \\
\hline & & size & 586 & 6723 & 878.7 & 557.1 \\
\hline
\end{tabular}

\subsection{Dataset}

Using the IoTPOT [15], 54,544 specimens were collected from October 2, 2016 to January 20, 2018. We disassembled specimens and used 24,126 cases whose main function was identified. For specimens whose main function was unidentified, it is highly probable that the function name has been deleted or the file has been corrupted. Therefore, such specimens were excluded from the analysis.

Table 3 shows a breakdown of the malware family names and ISAs (Instruction Set Architectures) acquired by the Linux readelf command [12] and AVClass [17]. Here, specimens whose malware family name is NaN were not uploaded to VirusTotal [18] during data acquisition. In addition, SINGLETON is a specimen whose AVClass could not determine a malware family name for various reasons, e.g., all of VirusTotal's labels are generic.

We generated signature-FCSGs from source code of Bashlite and Mirai for this investigation, and extracted FCSGs from all specimens. The order and size of those FCSGs are summarised in Table 4, where order and size mean the number of nodes and that of edges in an FCSG. Table 4 shows Min., Max., Avg., and Std. denote minimum, maximum, average, and standard deviation, respectively. In the table, All means those of all specimens (i.e., 24,126 specimens). In addition to all specimens, Table 4 only shows the values for the Bashlite and Mirai specimens and omits the specimens categorized to the other families (e.g., Tsunami and Lightaidra). This is because the other families had the tendency similar to Bashlite and Mirai.

\subsection{Signature Matching Results}

Table 5 shows the number of Bashlite signature matches (in Table 1) and a breakdown of the malware family. Among the analysis targets, 20,026 malware were determined to be Bashlite by AVClass, and there existed 19,954 Bashlite specimens that matched one or more signatures. Similarly, Table 6 shows the number of Mirai signature matches (in Table 2) and a breakdown of the malware family. Here, 122 specimens were determined to be Mirai by AVClass, and one or more signatures matched 121 specimens. In addition, Tables 7 and 8 show the number of matched specimens for each signature.

5.2.1 Investigation 1: Specimens Mixed with Malware Families. As shown in Tables 5 and 6, we found that some specimens matched multiple signatures from different malware families. These are mixed variants created by adding some behaviors of specimens with different malware families. 
Table 5: How many Bashlite signatures out of 13 signatures were matched to specimens in the dataset (e.g., five Bashlite signatures were matched to 40 Tsunami specimens). The signatures are listed in Table 1.

\begin{tabular}{|c|c|c|c|c|c|c|c|c|}
\hline & \multicolumn{6}{|c|}{ Malware familiy } & \multirow[b]{2}{*}{ Total } \\
\hline & & $\begin{array}{l}\text { Bash- } \\
\text { lite }\end{array}$ & Mirai & $\begin{array}{l}\text { Tsu- } \\
\text { nami }\end{array}$ & $\begin{array}{l}\text { Light- } \\
\text { aidra }\end{array}$ & $\begin{array}{l}\text { SING- } \\
\text { LETON }\end{array}$ & $\mathrm{NaN}$ & \\
\hline \multirow{14}{*}{ 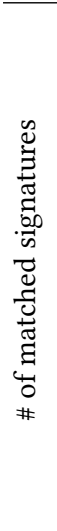 } & 0 & 57 & 121 & 5 & & & 68 & 251 \\
\hline & 1 & 13 & 1 & 268 & & & 61 & 343 \\
\hline & 2 & 193 & & 94 & & & 52 & 339 \\
\hline & 3 & 274 & & 10 & & 1 & 37 & 322 \\
\hline & 4 & 254 & & 36 & & 2 & 73 & 365 \\
\hline & 5 & 575 & & 40 & & 2 & 119 & 736 \\
\hline & 6 & 650 & & & 2 & & 167 & 819 \\
\hline & 7 & 587 & & & 2 & & 169 & 758 \\
\hline & 8 & 1327 & & & & & 452 & 1779 \\
\hline & 9 & 3277 & & & & & 584 & 3861 \\
\hline & 10 & 3410 & & & & & 528 & 3938 \\
\hline & 11 & 4432 & & & & & 628 & 5060 \\
\hline & 12 & 4798 & & & & & 548 & 5346 \\
\hline & 13 & 179 & & & & & 30 & 209 \\
\hline
\end{tabular}

Bashlite with Mirai behavior: As shown in Table 6, 119 Bashlite specimens matched the Mirai signature. These specimens matched two of Mirai's signatures, i.e., (main) init_hide_argv0, (main) init_hide_pname, or only (main) init_hide_argv0 (Table 8). When we decompiled and checked some of these specimens, their structure of basic blocks was that of Bashlite. Thus, these Bashlite specimens may have been generated with part of the Mirai source code to conceal the process name and argument.

Mirai with Bashlite behavior: As shown in Table 5, there was a single Mirai specimen that matched a Bashlite signature, and it was the signature copy_process in Table 7. However, the corresponding signature was not matched to the other Mirai specimens. Therefore, this single specimen is considered a Mirai variant, and it is possible that process duplication processing was added from the Bashlite source code.

In such cases, an analyst with prior knowledge may analyze the existence of multiple malware families' behavior. Therefore, it is expected that analysis can be performed while referencing the information of multiple malware families.

5.2.2 Investigation 2: Specialized Specimens for Some behaviors. As shown in Table 5, there were specimens whose Bashlite's signature was only partially matched. The following signatures were not matched when we conducted signature matching against those specimens.

- get_parameter

- get_ip

- get_mac

- exec_command

- sendHOLD

- sendJUNK

We decompiled those specimens and found that corresponding behaviors had been removed.From the above, it has been confirmed that there are simplified Bashlite variants produced by deleting some behaviors. In addition, the proposed method can detect the deletion of behavior in a malware specimen. In such cases, the
Table 6: How many Mirai signatures out of 15 signatures were matched to specimens in the dataset. The signatures are listed in Table 2.

\begin{tabular}{|c|c|c|c|c|c|c|c|c|}
\hline & \multicolumn{6}{|c|}{ Malware familiy } & \multirow[b]{2}{*}{ Total } \\
\hline & & $\begin{array}{c}\text { Bash- } \\
\text { lite }\end{array}$ & Mirai & $\begin{array}{l}\text { Tsu- } \\
\text { nami }\end{array}$ & $\begin{array}{l}\text { Light- } \\
\text { aidra }\end{array}$ & $\begin{array}{l}\text { SING- } \\
\text { LETON }\end{array}$ & $\mathrm{NaN}$ & \\
\hline \multirow{14}{*}{ 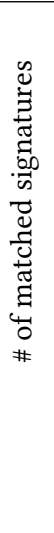 } & 0 & 19907 & 1 & 453 & 4 & 2 & 3404 & 23771 \\
\hline & 1 & 1 & 52 & & & & 22 & 75 \\
\hline & 2 & 118 & & & & 3 & 60 & 181 \\
\hline & 3 & & 2 & & & & 1 & 3 \\
\hline & 4 & & 13 & & & & 21 & 34 \\
\hline & 5 & & & & & & 1 & 1 \\
\hline & 6 & & 1 & & & & & 1 \\
\hline & 7 & & 23 & & & & 1 & 24 \\
\hline & 8 & & 12 & & & & 3 & 15 \\
\hline & 9 & & 15 & & & & 2 & 17 \\
\hline & 12 & & 2 & & & & 1 & 3 \\
\hline & 13 & & 1 & & & & & 1 \\
\hline & 14 & & & & & & & 0 \\
\hline & 15 & & & & & & & 0 \\
\hline
\end{tabular}

analyst may concentrate on analyzing existing behavior. Therefore, it is expected that rapid analysis will become possible.

5.2.3 Investigation 3: Specimens Matched with All Signatures. Some specimens matched all Bashlite signatures and most Mirai signatures. It is presumed that these specimens have nearly the same behavior group as the GitHub source code referenced when generating the signature. In such cases, nearly all source code is diverted when these specimens were created. Therefore, it is possible to respond quickly to malware based on the source code or existing analysis results.

5.2.4 Investigation 4: Specimens Unmatched with Any Signatures. Although specimens were determined to be Bashlite by AVClass, there were 57 specimens that did not match any Bashlite signatures. By investigating these specimens, it was confirmed that the program structure (i.e., the structure of basic blocks) differed from the released Bashlite source code [10]. As a result, the order of function calls changed significantly; therefore, although these specimens were determined as Bashlite, it is probable that the signatures did not match.

We also investigated one specimen determined to be Mirai by AVClass; however, none of the Mirai signatures were matched. When we checked the disassembled result, we found similar parts to both source codes (Bashlite [10] and Mirai [1]), but the structure for which the signature was created was different. Therefore, it appeared that the antivirus software detected the structural part of Mirai, and although the specimen was determined to be Mirai by AVClass, none of the signatures were matched.

Since it can be estimated that the specimens, as mentioned above, have a different structure from the referenced source code, it is necessary to analyze them as new specimens. However, once signatures are generated from such modified specimens, those signatures can be applied to other specimens whose structure is the same as or similar to that of those modified specimens. 
Table 7: The number of specimens matching each Bashlite signature

\begin{tabular}{|c|c|c|c|c|c|c|c|c|}
\hline & \multicolumn{6}{|c|}{ Malware familiy } & \multirow{2}{*}{ Total } \\
\hline & & Bashlite & Mirai & Tsunami & Lightaidra & SINGLETON & $\mathrm{NaN}$ & \\
\hline \multirow{13}{*}{ 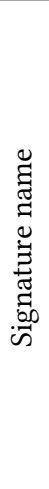 } & access_to_c2 & 17630 & & & 1 & 5 & 2540 & 20176 \\
\hline & echo_build_info & 11982 & & & 1 & & 1195 & 13178 \\
\hline & copy_process & 19721 & 1 & 447 & 4 & 5 & 3366 & 23544 \\
\hline & ping_pong & 19480 & & & 4 & 5 & 3295 & 22784 \\
\hline & exec_command & 5206 & & & & & 919 & 6125 \\
\hline & get_parameter & 13270 & & & 4 & 4 & 2265 & 15543 \\
\hline & get_ip & 17920 & & 147 & & & 3019 & 21086 \\
\hline & get_mac & 17658 & & 1 & & & 2925 & 20584 \\
\hline & get_random_str & 19224 & & 40 & 4 & 2 & 3195 & 22465 \\
\hline & sendHOLD & 8225 & & 33 & & & 1191 & 9449 \\
\hline & sendJUNK & 8194 & & 33 & & & 1181 & 9408 \\
\hline & sendTCP & 18454 & & 76 & 4 & & 3110 & 21644 \\
\hline & sendUDP & 19021 & & 53 & 4 & & 3173 & 22251 \\
\hline
\end{tabular}

Table 8: The number of specimens matching each Mirai signature (None of the signatures were matched to Tsunami and Ligtaidra)

\begin{tabular}{|c|c|c|c|c|c|}
\hline \multirow[b]{2}{*}{ Signature name } & \multicolumn{4}{|c|}{ Malware familiy } & \multirow[b]{2}{*}{ Total } \\
\hline & $\begin{array}{c}\text { Bash- } \\
\text { lite }\end{array}$ & Mirai & $\begin{array}{l}\text { SING- } \\
\text { LETON }\end{array}$ & $\mathrm{NaN}$ & \\
\hline init_argcheck & & 67 & & 29 & 96 \\
\hline init_hide_argv0 & 119 & 53 & 3 & 67 & 242 \\
\hline init_hide_pname & 118 & 53 & 3 & 67 & 241 \\
\hline init_print_system_exec & & 67 & & 30 & 97 \\
\hline initializer & & 67 & & 30 & 97 \\
\hline init_connect & & 1 & & & 1 \\
\hline scanner_init_init & & 3 & & 1 & 4 \\
\hline scanner_init_main & & 3 & & 1 & 4 \\
\hline attack_parse & & 31 & & 6 & 37 \\
\hline attack_start & & 54 & & 8 & 62 \\
\hline add_attack & & 121 & & 52 & 173 \\
\hline attack_app_http & & 17 & & 3 & 20 \\
\hline attack_app_cfnull & & 2 & & 1 & 3 \\
\hline attack_gre_ip & & 3 & & & 3 \\
\hline attack_gre_eth & & 3 & & & 3 \\
\hline
\end{tabular}

5.2.5 Investigation 5: Specimens in Other Malware Families. Table 7 shows that there existed Tsunami and Lightaidra specimens matching some Bashlite signatures. This indicates that they had the behaviors corresponding to the matched signatures of Bashlite. Table 8 also shows that Bashlite and SINGLETON were the only specimens that matched Mirai signatures. Considering this result, the source code of Mirai could not be often used to implement malware specimens except for Bashlite, whereas some Bashlite behaviors were implemented in multiple malware families.

Furthermore, it can be estimated that SINGLETONs, which could not be named by AVClass in this investigation, had both Bashlite and Mirai behavior. Therefore, even for such unknown specimens, the proposed method was able to identify some of their specific behaviors.

\section{RELATED WORK}

Relative to the function call sequence, Iwamoto et al. [9] extracted the call sequence of API functions after static analysis of Windows malware. Then they created a directed graph and calculated the similarity of the graph. Kikuchi et al. [13] generated API call graphs in the same manner reported in the literature [9], where common subgraphs were extracted between graphs. They proposed a method to measure the similarity between API call graphs and common subgraphs in order to cluster them and determine whether the specimens are unknown malware. In this paper, we extend their method [9] to include API calls and user-defined functions in the graph. We also add function name processing to make the influence of ISA less susceptible.

Focusing on malware signatures, Feng et al. [4] vectorized the control flow obtained by static analysis and collected the vectors generated from multiple ISA binaries. They then proposed a method to determine whether an unknown binary file contains a vulnerability by vectorizing the vulnerability part into a signature and matching vectors at high speed. Xu et al. [20] improved processing speed using a neural network based on the method [4]. Sathyanarayan et al. [16] extracted Windows API calls via static analysis and calculated the appearance frequency of important APIs in API calls. They proposed a signature creation method that can detect entire malware without being bound by the malware family. The above methods generate signatures automatically by some specific calculation; however, associating signatures with malware behavior is difficult. In the current study, we hope to create a signature that the analyst can easily associate with a behavior; therefore, the FCSG is adopted.

\section{CONCLUSION}

In this paper, we proposed a method for identifying behaviors of IoT malware across different ISAs. The key idea behind our method to absorb ISAs differences is to use signature-FCSG, based on the fact that the processing order of function calls is not affected by ISA differences. We then investigated 24,126 in-the-wild malware specimens captured by an IoTPOT system to show behavioral differences. We confirmed that some specimens were generated by reusing part 
of source code of Mirai and that of Bashlite, which was one of our key findings in this paper. For efficient malware analysis, analysts should keep it in mind that a target specimen to be analyzed could belong to more than one IoT malware family; they could behave like Mirai and sometimes like Bashlite, for example.

As a drawback of the proposed method, it is necessary to manually generate signature-FCSGs. In our future work, we plan to consider a way to support generating signature-FCSGs. As another future work, we aim to investigate evolutionary relationships of malware variants. To this end, we plan to apply FCSGs to a phylogenetic tree of malware generated by the method of [7]. Currently, method [7] can divide malware specimens into clusters based on the generated tree; however, it does not show behavioral differences between malware specimens in the same cluster. With FCSGs, we hope that their behavioral differences will be uncovered in detail.

\section{ACKNOWLEDGMENTS}

The authors would like to thank the IoTPOT team at Yoshioka Laboratory of Yokohama National University for providing the IoT malware. This work was partially supported by the commissioned research by National Institute of Information and Communications Technology (NICT), JAPAN, and by JSPS KAKENHI Grant Number JP18H03291.

\section{REFERENCES}

[1] anthonygtellez. 2019. GitHub - anthonygtellez/BASHLITE: An archive of BASH LITE source code. Retrieved December 11, 2019 from https:/github.com/ anthonygtellez/BASHLITE

[2] Manos Antonakakis, Tim April, Michael Bailey, Matt Bernhard, Elie Bursztein, Jaime Cochran, Zakir Durumeric, J. Alex Halderman, Luca Invernizzi, Michalis Kallitsis, Deepak Kumar, Chaz Lever, Zane Ma, Joshua Mason, Damian Menscher Chad Seaman, Nick Sullivan, Kurt Thomas, and Yi Zhou. 2017. Understanding the Mirai Botnet. In 26th USENIX Security Symposium, USENIX Security 2017, Vancouver, BC, Canada, August 16-18, 2017. 1093-1110.

[3] L. P. Cordella, P. Foggia, C. Sansone, and M. Vento. 2004. A (sub)graph isomorphism algorithm for matching large graphs. IEEE Transactions on Pattern Analysis and Machine Intelligence 26, 10 (2004), 1367-1372.

[4] Qian Feng, Rundong Zhou, Chengcheng Xu, Yao Cheng, Brian Testa, and Heng Yin. 2016. Scalable Graph-Based Bug Search for Firmware Images (CCS '16). 480-491.

[5] Sri Shaila G, Ahmad Darki, Michalis Faloutsos, Nael Abu-Ghazaleh, and Manu Sridharan. 2019. IDAPro for IoT Malware analysis?. In 12th USENIX Workshop on Cyber Security Experimentation and Test (CSET 19).

[6] Aric A. Hagberg, Daniel A. Schult, and Pieter J. Swart. 2008. Exploring Network Structure, Dynamics, and Function using NetworkX. , 11 - 15 pages.

[7] Tianxiang He, Chansu Han, Ryoichi Isawa, Takeshi Takahashi, Shuji Kijima, Jun'ichi Takeuchi, and Koji Nakao. 2019. A Fast Algorithm for Constructing Phylogenetic Trees with Application to IoT Malware Clustering. In Neural Information Processing. Springer International Publishing, 766-778.

[8] Hex-Rays SA. 2020. IDA Pro - Hex Rays. Retrieved August 12, 2020 from https://www.hex-rays.com/products/ida/

[9] Kazuki Iwamoto and Katsumi Wasaki. 2012. Malware Classification Based on Extracted API Sequences Using Static Analysis. In Proceedings of the Asian Internet Engineeering Conference (AINTEC '12). 31-38.

[10] jgamblin. 2019. GitHub - jgamblin/Mirai-Source-Code: Leaked Mirai Source Code for Research/IoC Development Purposes. Retrieved December 11, 2019 from https://github.com/jgamblin/Mirai-Source-Code

[11] JM Project. 2019. JM Index (LDP man-pages) (Japanese). Retrieved December 11, 2019 from https://inuxjm.osdn.jp/INDEX/ldp.html

[12] Michael Kerrisk. 2019. readelf(1) - Linux manual page. Retrieved December 14, 2019 from http://man7.org/linux/man-pages/man1/readelf.1.html

[13] Hisashi Kikuchi and Takao Okubo. 2019. Malware Classification Method using Common API Call Graph. Symposium on Cryptography and Information Security (2019).

[14] McAfee Labs. 2020. McAfee Labs COVID-19 Threats Report, July 2020. Retrieved August 13, 2020 from https://www.mcafee.com/enterprise/en-us/assets/reports/ rp-quarterly-threats-july-2020.pdf
[15] Yin Minn Pa Pa, Shogo Suzuki, Katsunari Yoshioka, Tsutomu Matsumoto, Takahiro Kasama, and Christian Rossow. 2015. IoTPOT: Analysing the Rise of IoT Compromises. In 9th USENIX Workshop on Offensive Technologies (WOOT 15).

[16] V. Sai Sathyanarayan, Pankaj Kohli, and Bezawada Bruhadeshwar. 2008. Signature Generation and Detection of Malware Families. In Information Security and Privacy. 336-349.

[17] Marcos Sebastián, Richard Rivera, Platon Kotzias, and Juan Caballero. 2016. AVclass: A Tool for Massive Malware Labeling. In Research in Attacks, Intrusions, and Defenses. 230-253.

[18] VirusTotal. 2020. VirusTotal. Retrieved September 15, 2020 from https://www. virustotal.com/gui/

[19] Yang Xiao, Bihuan Chen, Chendong Yu, Zhengzi Xu, Zimu Yuan, Feng Li, Binghong Liu, Yang Liu, Wei Huo, Wei Zou, and Wenchang Shi. 2020. MVP: Detecting Vulnerabilities using Patch-Enhanced Vulnerability Signatures. In 29th USENIX Security Symposium, USENIX Security 2020, August 12-14, 2020. 11651182.

[20] Xiaojun Xu, Chang Liu, Qian Feng, Heng Yin, Le Song, and Dawn Song. 2017. Neural Network-based Graph Embedding for Cross-Platform Binary Code Similarity Detection. Proceedings of the 2017 ACM SIGSAC Conference on Computer and Communications Security (Oct 2017). 